\title{
Depolarisation and the study of galaxy cluster magnetic fields with the SKA
}

\author{
Martin Krause*, Paul Alexander, Rosie Bolton \\ Cavendish Laboratory, University of Cambridge \\ JJ Thomson Avenue, Cambridge CB3 OHE \\ E-mail: M.Krause@mrao.cam.ac.uk
}

\begin{abstract}
Future radio surveys, most notably with the SKA aim at determining the comological evolution of magnetic fields using Faraday rotation of a huge number of cosmological radio sources. In contrast to currently used techniques, these surveys are planned for sub-GHz radio frequencies. However, radio emission is typically depolarised at low frequencies due to differential Faraday rotation along different lines of sight within the telescope beam. Therefore, it is vital for the experiment to predict the exact frequency below which we expect depolarisation to occur.

We compare a new turbulent depolarisation model to the classical two-field case, finding many interesting similarities. We calculate the depolarisation frequency, below which magnetic field measurements become much more difficult for the new model. Extrapolating from recent data, we find a median depolarisation frequency of $300 \mathrm{MHz}$ for typical SKA sources, hence enabling the determination of magnetic fields via the SKA mid frequency band.
\end{abstract}

From Planets to Dark Energy: the Modern Radio Universe

October 1-5 2007

The University of Manchester, UK

${ }^{*}$ Speaker. 


\section{Introduction}

Magnetised media rotate the polarisation angle of light passing though them differently at different wavelengths; observations across a frequency band therefore allow determination of the Rotation Measure through the medium, which depends upon the magnetic field, the electron number density and the path length through the medium. Thus polarised sources behind galaxy clusters can be used to probe the Intra-cluster magnetic fields.

Recent Faraday studies of extragalactic radio sources have exploited mainly the high frequency capabilities of the present generation of radio telescopes [1, 国, also: Bryan Gaensler and Gabriele Giovannini, this proceedings], where the fractional polarisation is independent of frequency. Even at frequencies down to $1.4 \mathrm{GHz}$, depolarisation is usually not encountered [ 4 ]. The measurement of magnetic fields in clusters of galaxies is one of the key projects envisaged for the SKA [5, also: Paul Alexander, this proceedings]. The goal is to carry out the new surveys, with the SKA as well as precursor instruments at low frequencies $(0.3-1 \mathrm{GHz}$ is envisaged for the SKA), in order to do this simultaneously with high redshift HI surveys. Depolarisation could in principle be an issue here, but also has the potential to reveal information about unresolved scales. We have therefore updated the theory on depolarisation.

\section{Depolarisation: two-scale field}

A simple two-scale theory for magnetic fields has been established in the classical treatment by Burn [6]: Faraday depolarisation occurs, whenever the radio emission passes through a medium with non-uniform Faraday depth, which is not resolved by the telescope beam. In this situation, the differently rotated polarisation vectors add up incoherently, and the resulting polarisation oscillates around a few percent of the high frequency value. Burn has investigated the situation where the magnetic field along the line of sight can be split into a constant part on a scale $L$, and a random component, with Gaussian variance $2 H_{\mathrm{r}}^{2} / 3$ on a smaller scale $d$. For his representative slab case, the transition region of the fractional polarisation $P(v)$ can be characterised by the following equation:

$$
P(v)=P(\infty) \frac{1-\mathrm{e}^{-S}}{S}
$$

where $P(\infty)$ denotes the high frequency value, and $S$ is given by ${ }^{1}$ :

$$
S=\hat{\sigma}_{R M}^{2}(c / v)^{4}, \hat{\sigma}_{R M}=K n H_{\mathrm{r}} \sqrt{L d} .
$$

Finally, $n$ is the density of free electrons and $K$ the usual Faraday rotation constant. From this we calculate $v_{1 / 2}$, the frequency where the fractional polarisation drops to $50 \%$ of its high frequency value:

$$
v_{1 / 2}=\sqrt{\frac{\hat{\sigma}_{R M}}{23}} \mathrm{GHz}
$$

when $\hat{\sigma}_{R M}$ is measured in $\mathrm{rad} / \mathrm{m}^{2}$.

\footnotetext{
${ }^{1}$ In the approximation needed here, we neglect the oscillatory part.
} 

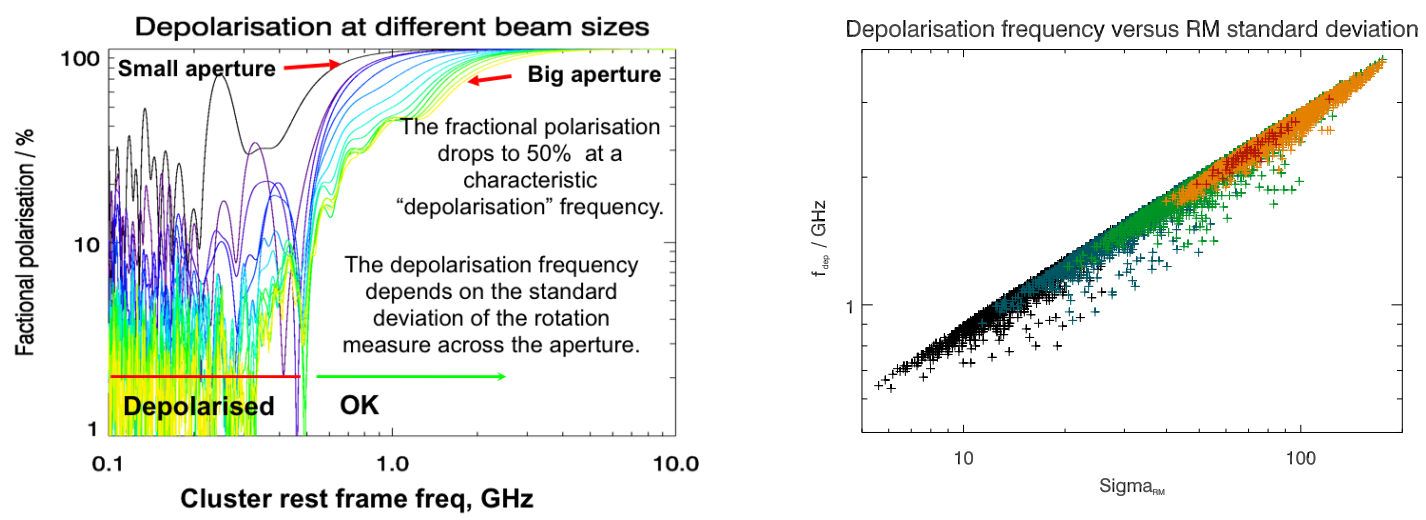

Figure 1: Left: Fractional polarisation over observing frequency for the turbulent field model. The different curves represent different aperture sizes. The curves are very similar to the two-scale case. Both show the steep drop-off at the depolarisation frequency and the oscillatory behaviour at lower frequencies. Right: Distribution of $v_{1 / 2}$ and $\sigma_{\mathrm{RM}}$ for many aperture sizes in a simulation cube. The different colours correspond to simulation cubes with different power spectra for the magnetic field.

\section{Depolarisation: turbulent field}

Turbulent fields have fluctuations on many scales; an analytic generalisation of the two-scale theory is therefore not straightforward. We have addressed this issue numerically, setting up a turbulent magnetic field in a computational box of up to $768^{3}$ cells, following the procedure described by [7]. The magnetic field is set up divergence free and with a Gaussian probability distribution function. The structure is characterised by the power spectrum, which is a power law: $k^{2}\left|B_{k}\right|^{2} \propto k^{-n}$. We use values for $n$ between zero (flat) and two, where $n=5 / 3$ corresponds to Kolmogorov turbulence. First, we investigate the behaviour of the fractional polarisation against frequency (Fig. 1). We find that the behaviour is practically identical to the two-scale case. The functional form at the transition region is very similar, so is the oscillatory behaviour and the amount of residual polarisation at low frequency. Therefore, it is still useful to to define a depolarisation frequency $v_{1 / 2}$, as above. This depolarisation frequency depends on the aperture size, contrary to the two-scale case. This is due to the increase in the standard deviation of the rotation measure within the aperture as the aperture size is increased, because turbulence typically has more power on larger scales. On the smallest scales this is accompanied by numerical resolution problems in our model, which is probably dominant for the smallest aperture curve in Fig. 11 (left).

For a simulation cube with a given power spectrum, we have measured $v_{1 / 2}$ and $\sigma_{\mathrm{RM}}$ for a large number of apertures at random positions. The resulting probability distribution functions are distributed as a lognormal function whose mean rises with aperture. This is different from Burn's two-scale model, where the statistics are Gaussian. We plot $v_{1 / 2}$ against $\sigma_{\mathrm{RM}}$ in Fig. 1 (right): the points cluster around a line $v_{1 / 2}=\sqrt{\frac{\sigma_{R M}}{13}} \mathrm{GHz}$, for all the power spectra investigated, with a very small spread. Though $\sigma_{\mathrm{RM}}$ cannot simply be identified with $\hat{\sigma}_{\mathrm{RM}}$, the result is clearly similar to the two-field case.

The difference between the power spectra consists in the different scaling behaviour of $\sigma_{\mathrm{RM}}$ with aperture size. Theoretically, one would expect a scale-free behaviour for scale-free turbulence. 
However, in a $768^{3}$ box, we can hardly expect one decade of scale-free behaviour. For Kolmogorov turbulence, we measure:

$$
\sigma_{\mathrm{RM}} \propto a^{0.8 \pm 0.2}
$$

where $\mathrm{a}$ is the aperture radius.

\section{Discussion}

Depolarisation has been measured for galactic and extragalactic radio sources by [B] and [ 9$]$. Extragalactic radio sources show a lognormal distribution of $v_{1 / 2}$ with a median around $3 \mathrm{GHz}$. This result is resolution dependent, and so the SKA may be expected to reach lower depolarisation frequencies.

Extrapolating the $\sigma_{\mathrm{RM}}=50 \mathrm{rad} / \mathrm{m}^{2}$ measured by Guidetti et al. [2] in a nearby cluster on scales of $100 \mathrm{kpc}$, we get with the above scaling laws, for Kolmogorov turbulence:

$$
v_{1 / 2}=0.3(a / \mathrm{kpc})^{0.4} \mathrm{GHz} \text {. }
$$

One kpc corresponds to about 0.1-0.2 arcsec at redshifts $>0.3$. For such clusters, half of the sources would remain polarised across the whole SKA mid frequency band (0.3-1 GHz), and for most sources, at least part of the frequency range could be used.

\section{References}

[1] C.L.Carilli, G.B.Taylor, 2002,40, 319 ARA\&A

[2] D.Guidetti et al., 2007, A\&A, in press, [astro-ph/0709.2652]

[3] J.Machalski, M.Jamrozy, 2006, A\&A 454,95

[4] J.Machalski, et al., 2006, A\&A 454,85

[5] B.M.Gaensler et al., 2004, New Astron. Rev., 48, 1003

[6] B.J.Burn, 1966, MNRAS, 133, 67

[7] M.Murgia et al., 2004, A\&A, 424, 429

[8] R.D.Davies, 1968, Nature,218,435

[9] L.Noreau, 1979, MSc thesis, University of Toronto 